This item was submitted to Loughborough's Research Repository by the author.

Items in Figshare are protected by copyright, with all rights reserved, unless otherwise indicated.

\title{
Contact detection and object size estimation using a modular soft gripper with embedded flex sensors
}

PLEASE CITE THE PUBLISHED VERSION

https://doi.org/10.1109/IROS.2018.8593399

PUBLISHER

(C) IEEE

VERSION

AM (Accepted Manuscript)

\section{PUBLISHER STATEMENT}

This work is made available according to the conditions of the Creative Commons Attribution-NonCommercialNoDerivatives 4.0 International (CC BY-NC-ND 4.0) licence. Full details of this licence are available at: https://creativecommons.org/licenses/by-nc-nd/4.0/

\section{LICENCE}

CC BY-NC-ND 4.0

\section{REPOSITORY RECORD}

Elgeneidy, Khaled, Gerhard Neumann, Simon Pearson, Michael R. Jackson, and Niels Lohse. 2019. "Contact Detection and Object Size Estimation Using a Modular Soft Gripper with Embedded Flex Sensors". figshare. https://hdl.handle.net/2134/34063. 


\title{
Contact Detection and Object Size Estimation using a Modular Soft Gripper with Embedded Flex Sensors*
}

\author{
Khaled Elgeneidy, Gerhard Neumann, Simon Pearson, Michael Jackson, and Niels Lohse
}

\begin{abstract}
Soft-grippers can grasp delicate and deformable objects without bruise or damage as the gripper can adapt to the object's shape. However, the contact forces are still hard to regulate due to missing contact feedback of such grippers. In this paper, a modular soft gripper design is presented utilizing interchangeable soft pneumatic actuators with embedded flex sensors as fingers of the gripper. The fingers can be assembled in different configurations using 3D printed connectors. The paper investigates the potential of utilizing the simple sensory feedback from the flex sensors to make additional meaningful inferences regarding the contact state and grasped object size. We study the effect of the grasped object size and contact type on the combined feedback from the embedded flex sensors of all fingers. Our results show that a simple linear relationship exists between the grasped object size and the final flex sensor reading at fixed input conditions, despite the variation in object weight and contact type. Additionally, by simply monitoring the time series response from the flex sensor, contact can be detected by comparing the response to the known free-bending response at the same input conditions. Furthermore, by utilizing the measured internal pressure supplied to the soft fingers, it is possible to distinguish between power and pinch grasps, as the nature of the contact affects the rate of change in the flex sensor readings against the internal pressure.
\end{abstract}

\section{INTRODUCTION}

Robotic manipulation of delicate and deformable targets is an increasingly sought-after research challenge that is motivated by diverse applications such as automated crop harvesting and food handling [1], safe human-robot interactions [2], sampling of sensitive species [3], and minimally invasive surgery [4]. To address this challenge, different soft robotic gripper designs have been proposed over the past few years that benefit from being inertly safe and passively compliant as outlined in recent reviews [5], [6]. Those soft grippers demonstrated interesting grasping capabilities, despite the simple materials and methods involved, which would have been rather challenging and more expensive to achieve with traditional rigid grippers [7]. The properties of a soft gripper are defined by the morphology of its fingers and their actuation method. Some soft grippers utilize well-established pneumatically actuated McKibben muscles as gripper fingers, which can be also combined with variable stiffness capability [8]. A more recent concept for softer gripper fingers is based on fluidic bending actuators, which are made from highly stretchable and soft silicone-rubber materials that can be easily

*Research supported by EPSRC.

K. Elgeneidy (mmkame@lboro.ac.uk), M. Jackson (m.r.jackson@lboro.ac.uk), and N. Lohse (N.Lohse@lboro.ac.uk) are with the Wolfson School of Mechanical, Electrical and Manufacturing Engineering, Loughborough University, United Kingdom. shaped following different morphologies [9]. This property has been demonstrated in many soft gripper examples, such as the RBO hand which is composed of a soft palm and three fiber-reinforced pneumatic actuators made from silicone rubber as the soft fingers [10]. More recently, 3D printing provided a faster and more automated fabrication process for soft grippers [11]. This process was utilized to print similar soft grippers based on soft fluidic actuators, including a highforce four-fingered soft gripper [12] and printed soft grippers with prestressed fingers for food handling applications [13]. Moreover, the phenomenon of particle-jamming was harnessed to create highly adaptable grippers [14]. A notable example is the universal soft gripper, which uses vacuum induced jamming of granular material inside a flexible membrane to grasp a wide range of objects [15]. The same particle-jamming principle has been applied instead to the fingertips of two-motor-controlled gripper fingers for lowcomplexity dexterous manipulation [16]. Furthermore, particle-jamming was also combined with fiber-reinforced soft pneumatic actuators to create a soft gripper with variable stiffness capability [17]. A different approach to creating soft gripper fingers is achieved through stretched elastomer membrane with pattered electrodes that are supplied with high voltage to gently grasp objects through electro-adhesion forces [18].

Despite being tailored for adaption to variation, relying solely on passive compliance of soft grippers has its limitations. The absence of sensing results in the lack of contact feedback. Moreover, information about the grasped target such as size or position remains unknown. Contact information would be of special importance when dealing with sensitive and deformable targets that can be easily bruised or marked by excessive contacts. Examples of which include: fresh fruits and vegetables, bakery products, biological tissues, or when interacting with humans or living organisms. Thus, it is crucial to create soft grippers that combine the desired benefits of passive compliance with sensor-guided methods that can ensure a safer and more deterministic grasp. Commercially available resistive flex sensors offer a thin and flexible sensing solution, which can be easy integrated within the strain limiting layer of soft pneumatic actuators, in order to change in resistance upon bending [19]. Those have been utilized for haptic identification of grasped objects using a three-fingered soft gripper, which processes readings from the embedded flex

S. Pearson (spearson@lincoln.ac.uk) is with the Lincoln Institute of AgriFood Technology, University of Lincoln, United Kingdom.

G. Neumann (gneumann@lincoln.ac.uk) is with the Lincoln Centre for Autonomous Systems, School of Computer Science, University of Lincoln, United Kingdom. 
sensors to distinguish between a variety of grasped objects [20]. In addition, calibrated measurements from embedded flex sensors were used to achieve accurate closed-loop control of soft cylindrical actuators [21] and ribbed soft actuators [22]. The same approach was also applied to 3D printed soft gripper fingers based on the pleated morphology to study their grasping response [23]

In this paper, we investigate using the feedback from flex sensors embedded in the fingers of a modular soft gripper to not only estimate the grasped object size, but also to detect contact with the target and infer the general grasp type. In our previous work, we showed that a single soft finger can be reliably modelled following a data-driven approach to estimate the bending angle based on the combined feedback from the embedded flex sensors and onboard pressure sensors [24]. However, this approach requires testing and calibrating each individual finger to generate the required experimental data, which could be time-consuming. In this work we demonstrate how combining the raw sensory feedback from opposing soft gripper fingers during grasp tests, can be directly utilized to (1) provide estimates for the grasped object size, (2) detect contact with the grasped object when compared to the known free bending response, and (3) infer whether the contact is at the fingertip (pinch grasp) or spread across the inside of the fingers (power grasp) using the measured internal pressure response.

The paper starts by briefly outlining the structure of the modular soft gripper design, utilizing interchangeable soft pneumatic actuators with embedded flex sensors. In the next section, we state details about the designed experiment for investigating the sensory response when grasping objects of different sizes and weights. The results are then presented for object size estimation, contact detection, and grasp type identification. We conclude with discussing how our findings could be used to improve the manipulation of delicate and deformable targets.

\section{MODULAR SOFT GRIPPER DESIGN}

We embedded the single soft pneumatic actuators with flex sensors. The single actuators were combined a using 3D printed flange and connectors to create a multi-fingered modular soft gripper that can be assembled with two, three, or four soft finger modules based on the application needs. The base of each soft finger is encapsulated in a 3D printed casing, which allows securing the soft finger to the main gripper flange. It also guides the pneumatic tubing and sensor wiring as shown in Figure 1. The pneumatic tubes are connected to the fingers through a needle puncturing the base of the internal fluidic channel to allow easy switching between different fingers. Such design maintains the modularity of the gripper, i.e., soft fingers of different lengths or materials can be easily swapped as required. Reconfiguring the soft gripper is achieved through the $3 \mathrm{D}$ printed flange that carries the soft finger modules in two, three, or four finger arrangements. Hence, the fingers can be easily swapped or rearranged depending on the application needs. Having more fingers is desirable for objects of complex geometries to achieve a more stable grasp, while less fingers are preferable when operating in confined spaces.

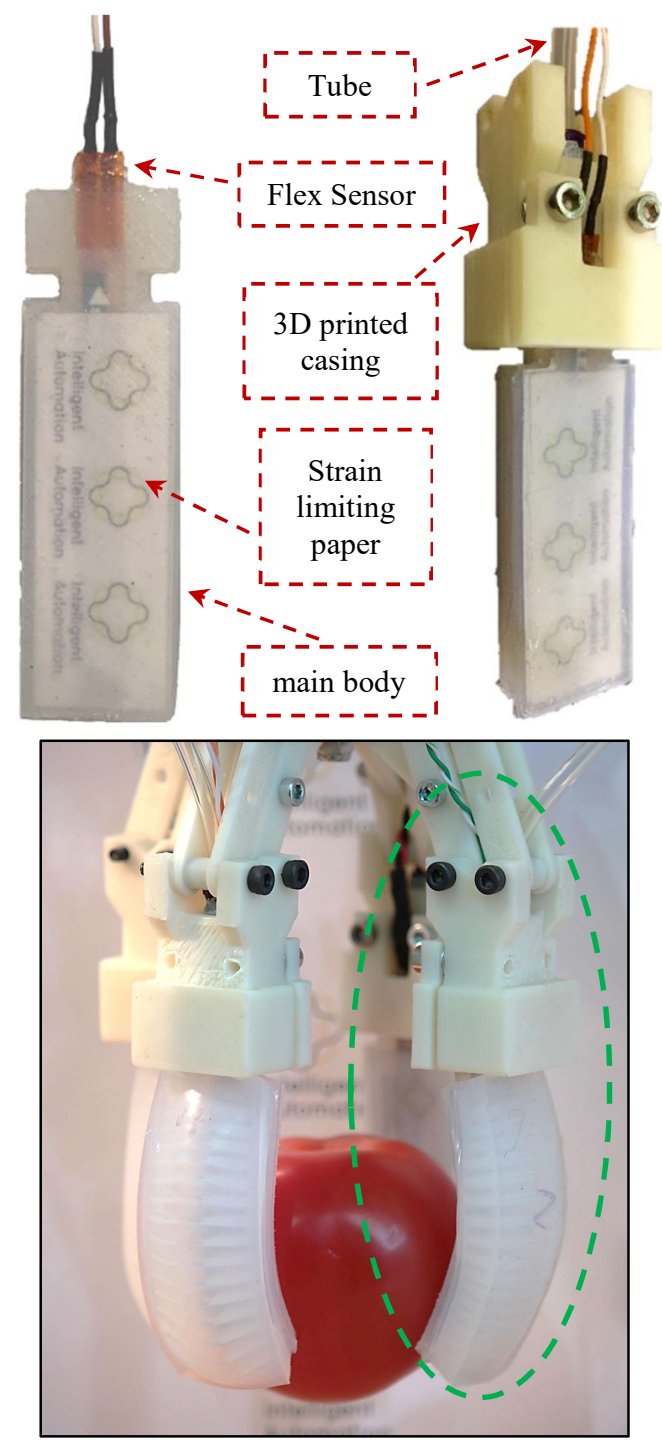

Figure 1: A reconfigurable soft gripper prototype consisting of four sensorised soft finger modules. (a) Individual soft finger showing the embedded flex sensor. (b) Printed casing added for routing wires and tube (c) Four soft fingers assembled together using 3D printed connectors gently grasping a tomato

The main advantages of our modular soft gripper design can be summarized as follows:

- The soft fingers can be easily replaced when damaged or to meet different application criteria, such as size and stiffness.

- The gripper can be assembled using 2, 3 or 4 soft fingers to account for the nature of the target object and the workspace limitations.

- The spacing between the fingers can be increased to enable grasping larger objects, or decreased in order to avoid excessive bending when grasping smaller objects using pinch grasps. 
- The 3D printed base can be easily customized to allow the gripper to be securely mounted to an existing robotic arm.

- It has a simple structure and is inexpensive to fabricate, compared to conventional rigid grippers with active compliance.

\section{EXPERIMENTAL DESIGN}

We designed our experiments to investigate the effect of variable object sizes and weights on the resulting feedback from the embedded flex sensors. We want to evaluate whether the sensor provides sufficient feedback for detecting contact with the grasped object and estimating its size. To achieve this, a set of spherical objects of different diameters and weights were prepared for our grasping experiments. The set included seven spheres with diameters of $50.8 \mathrm{~mm}$ (2") and $25.4 \mathrm{~mm}$ (1”), which were machined with high precision $( \pm 0.05 \mathrm{~mm})$ from different materials. Spheres of the same diameter have different weights due to the different material. Using spherical objects avoid affecting the results due to the variations in the grasped object orientation, i.e., the maximum distance between two opposing soft fingers will be always equal to the diameter of the grasped sphere. Additional test objects with different geometries and weights were also included for validation purposes. The different combinations of sizes and weights of the grasped objects are shown in Table 1.

TABLE 1: SUMMARY OF THE PROPERTIES OF THE GRASPED OBJECTS

\begin{tabular}{|c|c|c|c|}
\hline$\#$ & geometry & size $(\mathbf{m m})$ & weight $(\mathbf{g})$ \\
\hline 1 & sphere & 50.8 & 62 \\
\hline 2 & sphere & 50.8 & 81 \\
\hline 3 & sphere & 50.8 & 93 \\
\hline 4 & sphere & 50.8 & 143 \\
\hline 5 & sphere & 25.4 & 7 \\
\hline 6 & sphere & 25.4 & 9 \\
\hline 7 & sphere & 25.4 & 18 \\
\hline ping pong & sphere & 37.5 & 3 \\
\hline Tennis ball & sphere & 64.5 & 57 \\
\hline Bulb & Var curvature & $\sim 59$ & 28 \\
\hline block & Cuboid & 50.5 & 9 \\
\hline dice & cube & 39 & 29 \\
\hline
\end{tabular}

Each of those objects were grasped twice at the same input pressure of $10 \mathrm{Psi}(68.9 \mathrm{kPa})$. The resulting readings from each embedded flex sensor, as well as the measured internal pressure, were recorded for each test. Each opposing soft finger pairs were connected through the same pressure input, to allow them to reach an equilibrium position. Figure 2 shows sample images for some of the grasping tests. The soft gripper was able to successfully grasp all the objects throughout the testing procedure.

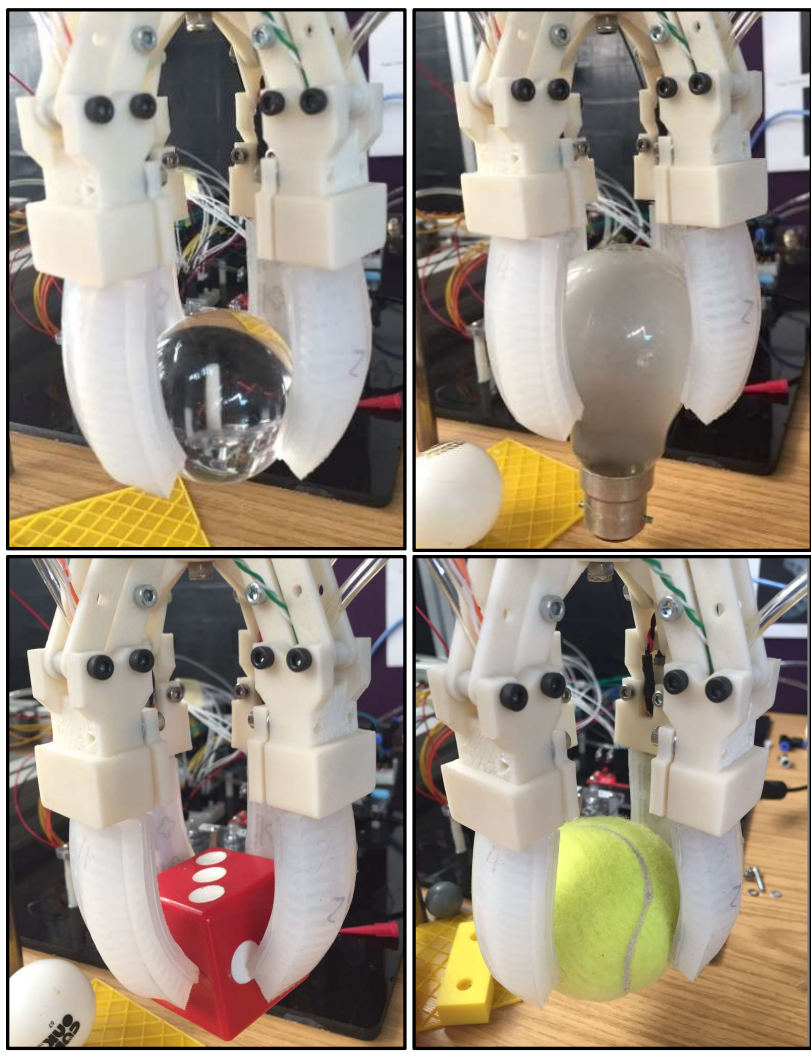

Figure 2: Sample images for grasping tests showing the soft gripper successfully grasping a small sphere, light bulb, dice and a tennis ball.

\section{RESULTS}

In this section, we will present our data analysis in terms of object-size estimation, contact detection and estimation of the grasp type.

\section{A. Object-Size Estimation}

In our first analysis, we want to investigate whether we can estimate the size of the grasped object from the sensor information provided by the flex sensors. Figure 3 shows the final values from the flex sensors embedded in two opposing soft fingers against the grasped object size. Taking the average value from the opposing soft fingers reduces the effect of random variance, which is demonstrated by the improved $\mathrm{R}^{2}$ value of 0.971 (compare to 0.957 and 0.915 for the individual sensors). A simple linear relationship is clear from the data acquired from 24 grasp tests on the 12 objects. We can see that the response from each sensor is noticeably different, since the sensor sensitivity and base resistance value is not identical for different sensor samples. Yet, the relationship remains consistent for each individual sensor across the grasp tests. Objects with same size are closely clustered around the same value with limited variance as to be expected from the difference in weight and contact type. We can also observe that test objects of different geometries still follow the same relationship, indicating that such a relationship can be extended to a wider range of object geometries, as long as the operating conditions are maintained. This would require a larger set of test objects to generate sufficient data. 


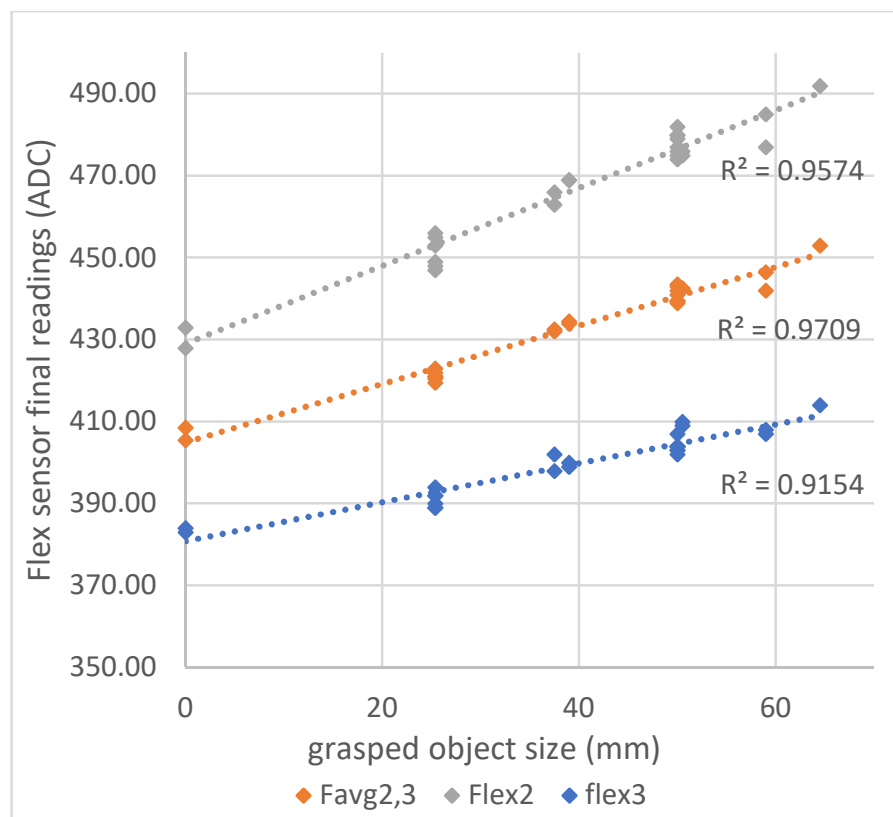

Figure 3: Final readings from embedded flex sensors against the grasped object size

\section{B. Contact Detection}

To assess the ability of contact detection, we show the time series responses for the flex sensors embedded in two opposing soft fingers when grasping the set of spheres (1 to 7) of sizes 50.8 and $25.4 \mathrm{~mm}$, as well as the free bending response at the same input pressure in Fig 4. Each grasping experiment was repeated twice, resulting in 8 response plots for the larger spheres and 6 response plots for the smaller size. We can observe three distinct response curves corresponding to the small $25.4 \mathrm{~mm}$ spheres, the larger $50.8 \mathrm{~mm}$ spheres, and the free bending response. This plot not only confirms the repeatability of the results acquired for grasping an object of a specific size at a fixed input pressure, but also illustrates how the time series response from the flex sensor varies when conforming to different sized objects. The steepest response is witnessed by the free bending case, as no external contact is limiting the soft fingers to bend. The other two responses start to deviate away from the free bending response at different points, with the smaller sphere response deviating later at a steeper rate of change. This effect is caused by the early contact that the soft fingers make with the larger spheres along the fingers' length, limiting the bending response early on while continuing to deform to conform to the grasped object surface. On the other hand, the response from the smaller sphere initially follows the free bending response, since the fingers are indeed freely bending when the actuation first starts, until they reach the small spheres. Once the fingertip makes contact with the spheres, the response will be restrained causing the witnessed deviation from the free bending response. Yet, the response does not completely stop at this point since the fingers are only constrained at the tip, so their bodies continue to deform, but with a slower rate, until reaching the final grasp profile.

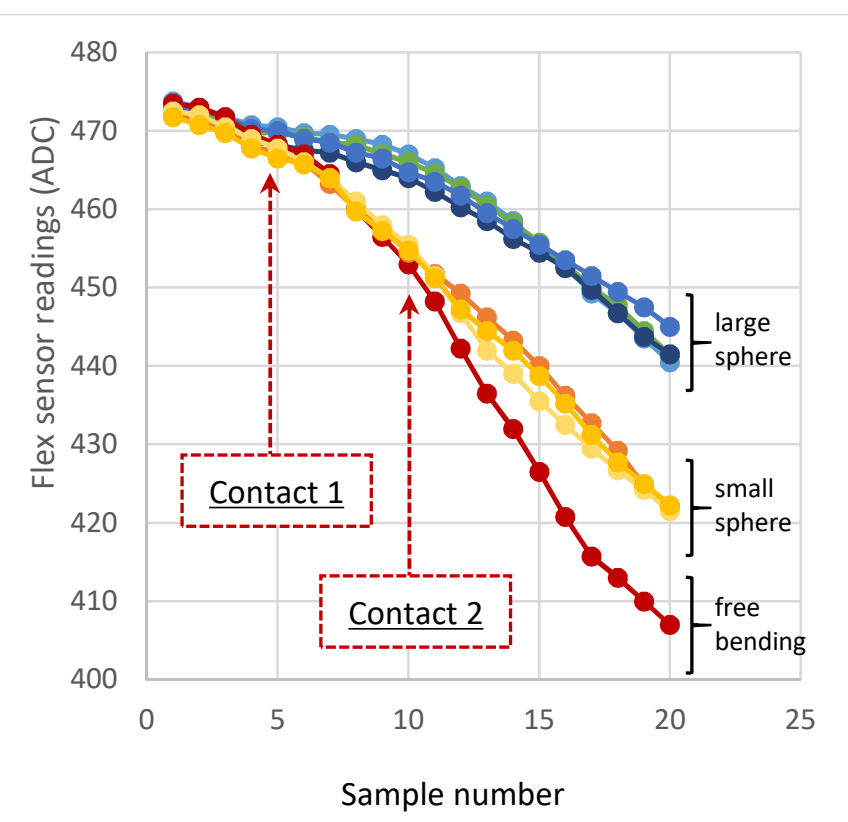

Figure 4: The averaged flex sensor response from opposing embedded flex sensors.

\section{Grasp Type Identification}

Figure 5 shows the average readings from opposing flex sensors against the supplied pressure when grasping each of the 7 spheres (balls 1 to 7 ) of sizes 25.4 and $50.8 \mathrm{~mm}$ twice.

A very interesting observation becomes evident, in which the response is divided into two distinct response curves. The tests involving the smaller spherical object all follow the same response curve, which is steeper than the one followed by all the larger spheres. This behavior is expected to be due to the difference in the contact type when grasping those two sizes of spheres. Smaller spheres are grasped at the fingertips of the gripper (pinch grasp), while the larger spheres are encapsulated within the gripper fingers with a larger contact area at the inside surface of the fingers (power grasp). Hence, by combining the internal pressure response to reflect the corresponding change in the flex sensor readings, it becomes possible to identify the general grasp type corresponding to how contact location with the fingers. This observation can be explained by the fact that power grasps generally involve a larger contact area with the inside surface of soft gripper fingers, which suppresses their bending deformation, while pinch grasps exhibit contacts at a limited area at the fingertip. Furthermore, the free bending response followed the same response curve of the smaller spheres, since in both cases the fingers are freely bending during most of the operation, with the latter case being stopped earlier when making contact with the small sphere at the fingertips. Hence, as long as the monitored response follows that of the known free bending response, then it can be assumed that no significant contact with the target object has been made yet. However, If the monitored response starts to deviate from the free bending response early on, then this signifies that the object is being encapsulated inside the gripper. Yet, the soft fingers will still continue to deform until fully conforming to the grasped object surface. 


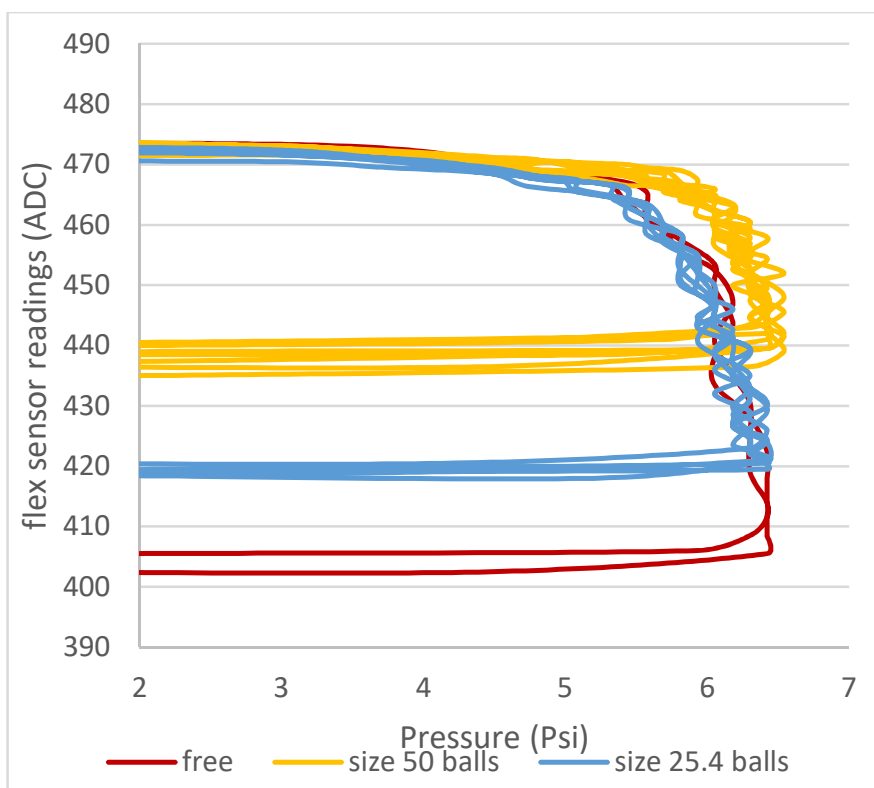

Figure 5: Flex sensor response against the measured internal pressure for small and large spheres as well as the free bending response

To validate those observations, each of the test objects were also grasped twice while recording the sensory response to be compared to the original two responses identified. The results are plotted in Error! Reference source not found., showing how the new responses fell within one of the previously identified response curves. It can be observed that the light bulb and tennis ball fell closely along the response curve by the larger spheres, which is expected since the diameters were in the same range and were grasped following a power grasp. On the other hand, the ping pong ball, dice, and cuboid block all closely followed the response by the smaller sized spheres. Again, the sizes of those objects were small and grasped following the pinch grasp at the fingertip. Thus, the final value reflects the object size as previously discussed, while the profile of the response curve reflects the grasp type.

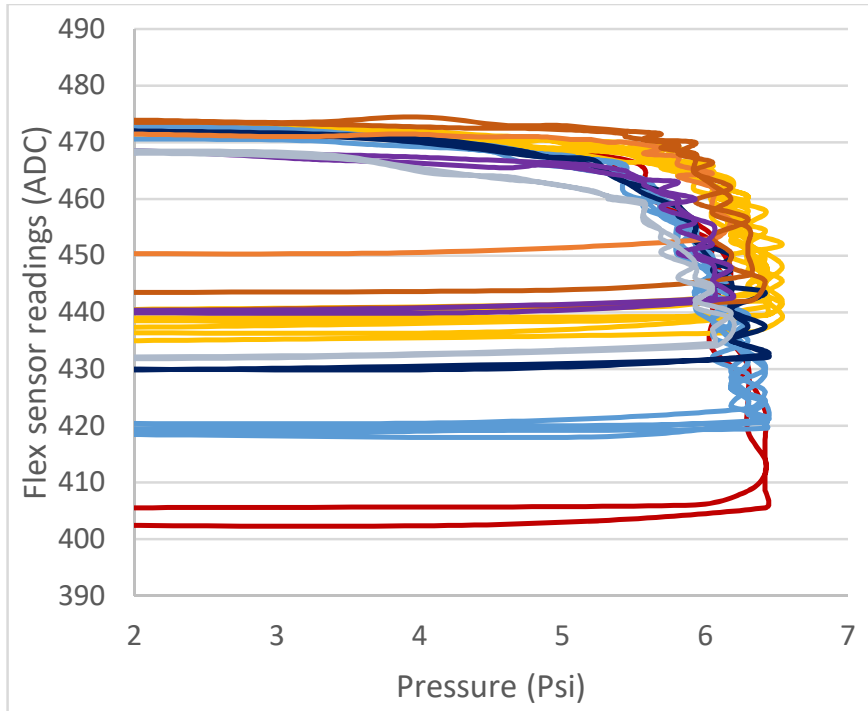

Figure 6: Flex sensor response against the measured internal pressure after adding the responses from test objects with variable geometries

\section{DISCUSSION}

The results of this work demonstrated new possibilities for further utilization of simple sensory feedback from soft gripper fingers to detect contact, estimate object size, and infer the general grasp type. The conducted experiment showed that a clear linear relationship exists between the average final value from the flex sensors embedded in opposing soft fingers, and the grasped object size. The relationship was consistent across all the grasped objects despite the variation in their sizes and weight. This indicates that the object weight had minimal influence on the readings which contributed to the Limited variance witnessed.

Furthermore, examining the time-series response of the embedded flex sensor during grasp tests showed that the rate of change of the response curve changes upon making contact with the grasped objects. All the smaller $25.4 \mathrm{~mm}$ spheres grasp tests followed the same response curve, which is steeper than that witnessed by all the larger $50.8 \mathrm{~mm}$ spheres. Comparing those to the free bending response shows that initially all responses follows the same path, with the response from the larger spheres deviating early on followed later by the deviation of smaller spheres response. This is expected to be due to the early contact that the soft fingers make with the larger objects as they get encapsulated within the gripper, causing the witnessed early deviation. Hence, three distinct responses can be clearly witnessed, which were consistent across all the reputations of the grasp tests. The importance of this observation lies in the fact that prior knowledge of the free bending response of the soft gripper can be utilized to detect contact with a target by simply monitoring the difference between the current response and the known free bending one at the same input pressure. Thus, once the difference between the current response and the known free-bending one exceeds a set threshold, it can be assumed that contact has occurred and the supplied pressure can be adjusted accordingly. This is essential for soft grippers handling delicate and sensitive targets, in order to avoid damaging or bruising them from excessive contacts.

Moreover, another interesting finding from this experiment became evident by plotting the response from the embedded flex sensors against the response from the internal pressure supplied to opposing soft fingers. The results demonstrated consistent cycles representing the actuation and retraction of the soft fingers, which can be clearly divided into two distinct response curves. The first included all the large objects that were encapsulated within the gripper (power grasp). While the second set included all the smaller objects that were grasped using the gripper fingertips (pinch grasp), as well as the free bending response. This is to be expected as grasping smaller objects includes a free-bending phase initially, which is suddenly stopped when making contact with the object. The clear difference however, is the fact that the free-bending response extends the furthest along the curve when compared to the responses from pinch grasps, since it is completely unrestrained. In fact, the response from each grasped object ends at a different point depending on its size, which was 
confirmed by the clear relationship between object size and the final flex sensor value. This interesting observation showed that combining the internal pressure response with the flex sensor response can allow differentiating between the general grasp types. Currently only pinch and power grasps were implemented in the tests considering the structure of the gripper. Yet, this highlights the potential of combining limited sensory feedback to infer additional information about the nature of the grasps that are not easy to measure directly for a soft gripper.

\section{CONCLUSION}

The results of this work demonstrated how simple sensory feedback from flex sensors embedded within the fingers of a modular soft gripper, can be harnessed to infer the contact state, grasped object size, and the general grasp type. This is achieved simply by comparing the current sensory feedback during grasp operations, to that of the previously recorded dree bending state at the same input conditions. Hence, uncalibrated sensory feedback can still provide meaningful grasp information, as it is compared to that from the free bending state. The final readings from opposing soft fingers are strongly related to the grasped object size, while the freebending timeseries response can be compared to that during grasping to detect when contact occurs with the target object. Incorporating the measured internal pressure supplied to the soft fingers with the flex sensor response, highlights the rate of finger bending per unit pressure, which was shown to differ significantly based on the general grasp type (pinch or power grasp). The additional grasp information demonstrated in this work can be hence used to control the supplied pressure to a soft gripper to avoid excessive contact, which is of special importance to applications involving interacting with delicate targets such as fresh fruits and vegetables, bakery products, biological tissues, or living organisms.

Current planned work include extending the experiment using a wider set of objects with more variation in their geometry, to investigate the effect of the grasped object profile on the combined flex sensor response. To achieve this, a customized multi-segment flex sensor is being developed to enable a unique change in resistance that can better sense the deformed profile of a soft finger. Additionally, the sensor is to combined with a higher resolution $\mathrm{ADC}$, to enable finer changes in the flex sensor's resistance to be captured, which would be essential to detect the influence of grasped object geometry and weight. Nevertheless, the results of this work provide an important contribution to the adoption of sensor-guided methods to soft grippers, using simple sensors and gripper design. Not only can the size of grasped object be successfully estimated, but also further inferences can be made regarding the grasp type and contact detection. All of which are important grasp characteristics that would enable safer and more controllable soft grippers.

\section{ACKNOWLEDGMENT}

The reported work has been partially funded by the EPSRC Centre for Innovated Manufacturing in Intelligent Automation (EP/IO33467/1). The support of which is gratefully acknowledged.

\section{REFERENCES}

N. Kellaris, V. Gopaluni Venkata, G. M. Smith, S. K. Mitchell, and C. Keplinger, "Peano-HASEL actuators: Muscle-mimetic, electrohydraulic transducers that linearly contract on activation," Sci. Robot., vol. 3, no. 14, p. eaar3276, 2018.

P. Polygerinos, N. Correll, S. A. Morin, B. Mosadegh, C. D. Onal, K. Petersen, M. Cianchetti, M. T. Tolley, and R. F. Shepherd, "Soft Robotics: Review of Fluid-Driven Intrinsically Soft Devices; Manufacturing, Sensing, Control, and Applications in HumanRobot Interaction," Adv. Eng. Mater., pp. 1-22, 2017.

[3] K. C. Galloway, K. P. Becker, B. Phillips, J. Kirby, S. Licht, D. Tchernov, R. J. Wood, and D. F. Gruber, "Soft Robotic Grippers for Biological Sampling on Deep Reefs," Soft Robot., vol. 3, no. 1, pp. 23-33, 2016.

[4] M. Cianchetti, T. Ranzani, G. Gerboni, T. Nanayakkara, K. Althoefer, P. Dasgupta, and A. Menciassi, "Soft Robotics Technologies to Address Shortcomings in Today's Minimally Invasive Surgery: The STIFF-FLOP Approach," Soft Robot., vol. 1, no. 2, pp. 122-131, 2014.

[5] J. Hughes, U. Culha, F. Giardina, F. Günther, and A. Rosendo, "Soft Manipulators and Grippers: A Review," Front. Robot. AI, vol. 3, no. November, pp. 1-12, 2016.

[6] K. Tai, A.-R. El-Sayed, M. Shahriari, M. Biglarbegian, and S. Mahmud, "State of the Art Robotic Grippers and Applications," Robotics, vol. 5, no. 2, p. 11, 2016.

[7] C. Laschi, B. Mazzolai, and M. Cianchetti, "Soft robotics: Technologies and systems pushing the boundaries of robot abilities," Sci. Robot., vol. 3690, no. 1, p. eaah3690, 2016.

[8] L. A. T. Al Abeach, S. Nefti-Meziani, and S. Davis, "Design of a Variable Stiffness Soft Dexterous Gripper," Soft Robot., vol. 0, no. 0, p. soro.2016.0044, 2017.

[9] A. D. Marchese, R. K. Katzschmann, and D. Rus, "A Recipe for Soft Fluidic Elastomer Robots," Soft Robot., vol. 2, no. 1, pp. 725,2015

[10] R. Deimel and O. Brock, "A compliant hand based on a novel pneumatic actuator," Proc. - IEEE Int. Conf. Robot. Autom., pp. 2047-2053, 2013.

[11] A. Zolfagharian, A. Z. Kouzani, S. Y. Khoo, A. A. A. Moghadam, I. Gibson, and A. Kaynak, "Evolution of 3D printed soft actuators," Sensors Actuators, A Phys., vol. 250, pp. 258-272, 2016.

[12] H. K. Yap, H. Y. Ng, and C.-H. Yeow, "High-Force Soft Printable Pneumatics for Soft Robotic Applications," Soft Robot., vol. 3, no. 3, pp. 144-158, 2016.

[13] Z. Wang, Y. Torigoe, and S. Hirai, "A Prestressed Soft Gripper: Design, Modeling, Fabrication, and Tests for Food Handling," IEEE Robot. Autom. Lett., vol. 2, no. 4, pp. 1909-1916, 2017.

[14] H. M. Jaeger, “Celebrating Soft Matter's 10th Anniversary: Toward jamming by design.," Soft Matter, vol. 11, no. 1, pp. 1227, Dec. 2014.

[15] E. Brown, N. Rodenberg, J. Amend, A. Mozeika, E. Steltz, M. R. Zakin, H. Lipson, and H. M. Jaeger, "Universal robotic gripper based on the jamming of granular material," Proc. Natl. Acad. Sci., vol. 107, no. 44, pp. 18809-18814, Oct. 2010.

[16] J. Amend and H. Lipson, "The JamHand: Dexterous Manipulation with Minimal Actuation," Soft Robot., vol. 4, no. 1, p. soro.2016.0037, 2017.

[17] Y. Wei, Y. Chen, T. Ren, Q. Chen, C. Yan, Y. Yang, and Y. Li, "A Novel, Variable Stiffness Robotic Gripper Based on Integrated Soft Actuating and Particle Jamming," Soft Robot., vol. 0, no. 0, p. soro.2016.0027, 2016.

[18] J. Shintake, S. Rosset, B. Schubert, D. Floreano, and H. Shea, "Versatile soft grippers with intrinsic electroadhesion based on multifunctional polymer actuators," Adv. Funct. Mater., pp. 1-28, 
2015.

[19] G. Saggio, F. Riillo, L. Sbernini, and L. R. Quitadamo, "Resistive flex sensors: a survey," Smart Mater. Struct., vol. 25, no. 1, p. $13001,2016$.

[20] B. S. Homberg, R. K. Katzschmann, M. R. Dogar, and D. Rus, "Haptic identification of objects using a modular soft robotic gripper," in IEEE International Conference on Intelligent Robots and Systems, 2015, vol. 2015-Decem, pp. 1698-1705.

[21] G. Gerboni, A. Diodato, G. Ciuti, M. Cianchetti, and A. Menciassi, "Feedback control of soft robot actuators via commercial flex bend sensors," IEEE/ASME Trans. Mechatronics, vol. 22 , no. 4, pp. 1-1, 2017.

[22] K. Elgeneidy, N. Lohse, and M. Jackson, "Bending angle prediction and control of soft pneumatic actuators with embedded flex sensors - A data-driven approach," Mechatronics, 2017.

[23] Z. Wang and S. Hirai, "A 3D printed soft gripper integrated with curvature sensor for studying soft grasping," in SII 2016 - 2016 IEEE/SICE International Symposium on System Integration, 2017, pp. 629-633.

[24] K. Elgeneidy, N. Lohse, and M. Jackson, "Bending angle prediction and control of soft pneumatic actuators with embedded flex sensors - A data-driven approach,” Mechatronics, Oct. 2017. 